\title{
Respiratory Rate Derived from Pulse Photoplethysmographic Signal by Pulse Decomposition Analysis
}

\author{
Jesús Lázaro, Member, IEEE, Spyridon Kontaxis, Raquel Bailón, Pablo Laguna, Fellow, IEEE and Eduardo Gil
}

\begin{abstract}
A novel technique to derive respiratory rate from pulse photoplethysmographic (PPG) signals is presented. It exploits some morphological features of the PPG pulse that are known to be modulated by respiration: amplitude, slope transit time, and width of the main wave, and time to the first reflected wave. A pulse decomposition analysis technique is proposed to measure these features. This technique allows to decompose the PPG pulse into its main wave and its subsequent reflected waves, improving the robustness against noise and morphological changes that usually occur in long-term recordings. Proposed methods were evaluated with a data base containing PPG and plethysmography-based respiratory signals simultaneously recorded during a paced-breathing experiment. Results suggests that normal ranges of spontaneous respiratory rate $(0.1-0.5 \mathrm{~Hz})$ can be accurately estimated (median and interquartile range of relative error less than 5\%) from PPG signals by using the studied features.
\end{abstract}

\section{INTRODUCTION}

Respiratory rate is a sensitive clinical parameter in a multitude of pulmonary diseases [1], e.g., it remains the first and often the most sensitive marker for acute respiratory dysfunction [2]. Respiratory rate is also useful for detection of periodic breathing [3] which results in elevated mortality in heart failure patients [4]. Furthermore, respiratory rate is currently getting more interest in the field of sports training monitoring [5]. Respiratory rate is usually measured by spirometry, pneumography, or plethysmography techniques, requiring cumbersome devices which may interfere with natural breathing. The disadvantages of these devices become more relevant in some applications such as sleep studies and stress test. Thus, some alternatives have been proposed in the literature. Some of them are based on the pulse photoplethysmographic (PPG) signal.

PPG signal is usually obtained from a fingertip by a comfortable and economical sensor composed of a few opto-

\footnotetext{
*This work has received funding from the European Union's Framework Programme for Research and Innovation Horizon 2020 (2014-2020) under the Marie Skodowska-Curie under Grant 745755, from the Ministerio de Economía y Competitividad under Project TIN2014-53567-R, from the Goverment of Aragón and European Social Fund (EU) through BSICoS group (T96), and from CIBER in Bioengineering, Biomaterials, and Nanomedicine (CIBER-BBN) through Instituto de Salud Carlos III. The computation was performed by the ICTS 'NANBIOSIS', more specifically by the High Performance Computing Unit of the CIBER in Bioengineering, Biomaterials \& Nanomedicne (CIBER-BBN) at the University of Zaragoza

J. Lázaro, S. Kontaxis, R. Bailón, S. Kontaxis, P Laguna and E. Gil are with the Communications Technology Group, I3A, University of Zaragoza and CIBER de Bioingeniería, Biomateriales y Nanomedicina (CIBER-BBN), Spain, \{jlazarop, skontaxis, rbailon, laguna, edugilh $\}$ at unizar.es

J. Lázaro is with the Department of Biomedical Engineering, University of Connecticut, Storrs, CT 06269, USA jesus.lazaro at uconn.edu
}

electric components, making it very interesting for ambulatory scenarios. Vital signs that can be obtained from the PPG signals include arterial blood oxygen saturation, pulse rate, and respiratory rate. However, most of the methods for deriving respiratory rate from PPG signal rely on morphological features which are very affected by noise and morphological changes that usually occur in long-time recordings.

Pulse decomposition analysis (PDA) aims to improve the robustness of different morphological measurements of the PPG pulse. It consists of modeling the PPG pulse as a superposition of different waves under the hypothesis of that it is formed by a main wave superposed with several reflected waves. Different models have been used in the literature, including a superposition of up to 5 Gaussians [6], and also other functions such as LogNormal [7] and Rayleight [8].

In this work, a modification of the PDA technique presented in [9] which assumes a model based on a superposition of Gaussian waves is used. The most particular characteristic of this PDA technique is that each wave is fitted separately, trying to impose physiology-related restrictions. Different morphological features of the PPG pulses which are expected to be modulated by respiration are measured from the parameters of the model, and derived respiration (DR) signals are obtained from them. Then, respiratory rate is estimated from these DR signals and compared to the respiratory rate measured by a plethysmography-based reference respiratory signal.

\section{METHODS}

\section{A. Signal acquisition and preprocessing}

Plethysmography-based respiration and PPG signals were recorded by Poly4 and Poly10 (Medicom MTD, Russia) using a sampling rate of $250 \mathrm{~Hz}$, from 17 healthy volunteers (12 men) who underwent to a breathing experiment. Subjects were visually guided to breathe at different constant rates: $0.1,0.2,0.3,0.4,0.5$, and $0.6 \mathrm{~Hz}$. Each one of the rates was requested during 2 minutes. For further analysis, data were split into segments of $60 \mathrm{~s}$ that were shifted every $10 \mathrm{~s}$.

A low-pass filter with a cut-off frequency of $35 \mathrm{~Hz}$ was applied to the PPG signals. Subsequently, the apex $\left(n_{\mathrm{A}_{i}}\right)$ and basal $\left(n_{\mathrm{B}_{i}}\right)$ points of the $i$ th PPG pulse $\left(x_{\mathrm{PPG}_{i}}(n)\right)$ were detected by an algorithm based on a low-pass-derivative filter and a time-varying threshold described in [10].

\section{B. Pulse decomposition analysis}

In order to apply a PDA technique, the beginning and end of the PPG pulses have to be determined. In this work, the $i$ th PPG pulse was considered to start at $n_{\mathrm{B}_{i}}$ and to end at $n_{\mathrm{B}_{i+1}}$. 
The baseline was estimated by cubic-splines interpolation of the PPG values at $n_{\mathrm{B}_{i}}$ and then, this estimated baseline was subtracted from the PPG signal.

The PDA technique presented in this work is based on [9]. Most of the PDA techniques try to fit a model based on a superposition of waves at once, sometimes leading to a physiologically unexpected decomposition such as a too small main wave. In difference, in this work, the fitting is performed in one step per modeled wave trying to impose restrictions related to physiology. Considering the first upslope of the PPG pulse to be mainly related to the upslope of the main wave, the first relative maximum $n_{\mathrm{A}_{i}}^{*}$ was detected. Thus, the first Gaussian of the model is fitted using only this PPG pulse first upslope. In order to do this fitting, a wave was generated by the first PPG pulse upslope followed by the same pulse upslope inverted in time:

$$
y_{1, i}(n)=x_{1, i}(n)+x_{1, i}\left(-n+2 n_{\mathrm{A}_{i}}^{*}+1\right),
$$

where $x_{1, i}(n)$ is the first upslope of the $i$ th PPG pulse, i.e., the PPG signal in the interval $\left[n_{\mathrm{B}_{i}}, n_{\mathrm{A}_{i}}^{*}\right]$. Then, $y_{1, i}(n)$ is normalized to the unit in amplitude and to 1000 samples in time. This normalized version is denoted $y_{1, i}^{r}(n)$, indicating that it is re-scaled. Subsequently, a Gaussian is fitted to it:

$$
\hat{y}_{1, i}^{r}(n)=A_{1, i}^{r} e^{\frac{-\left(n-B_{1, i}^{r}\right)^{2}}{C_{1, i}^{r}{ }^{2}}},
$$

where $A_{1, i}^{r}, B_{1, i}^{r}$, and $C_{1, i}^{r}$ are the amplitude, mean, and standard deviation of the Gaussian, respectively.

Then, a version of $\hat{y}_{1, i}^{r}(n)$ in the original amplitude and time scales was obtained and denoted $\hat{y}_{1, i}(n)$. This version was obtained by correcting the coefficients as:

$$
\begin{aligned}
A_{1, i} & =A_{1, i}^{r} \max \left\{y_{1, i}(n)\right\} \\
B_{1, i} & =B_{1, i}^{r} \frac{N_{1, i}}{1000} \\
C_{1, i} & =C_{1, i}^{r} \frac{N_{1, i}}{1000},
\end{aligned}
$$

where $N_{1, i}$ is the number of samples of $y_{1, i}(n)$.

Subsequently, a residual $s(n)$ was obtained as:

$$
s(n)=x_{\mathrm{PPG}_{i}}(n)-\hat{y}_{1, i}(n),
$$

where $x_{\mathrm{PPG}_{i}}(n)$ denotes the $i$ th PPG pulse.

The first reflected wave was obtained from $s(n)$. Similarly to the first step, the upslope of this wave is used to fit the rising part of a Gaussian, following an analogous procedure including the concatenation with the time-inverted upslope (similar to (1)), the amplitude and time normalization, the Gaussian fitting (similar to (2)), and the coefficient correction (similar to (3), (4), and (5)). In this case, the upslope is considered to end at the absolute maximum of $s(n)$, and to begin at its previous zero crossing. An illustration of the steps of this PDA technique can be observed in Fig. 1, and the same PPG pulse with its modeled main and first reflected waves can be observed in Fig. 2.
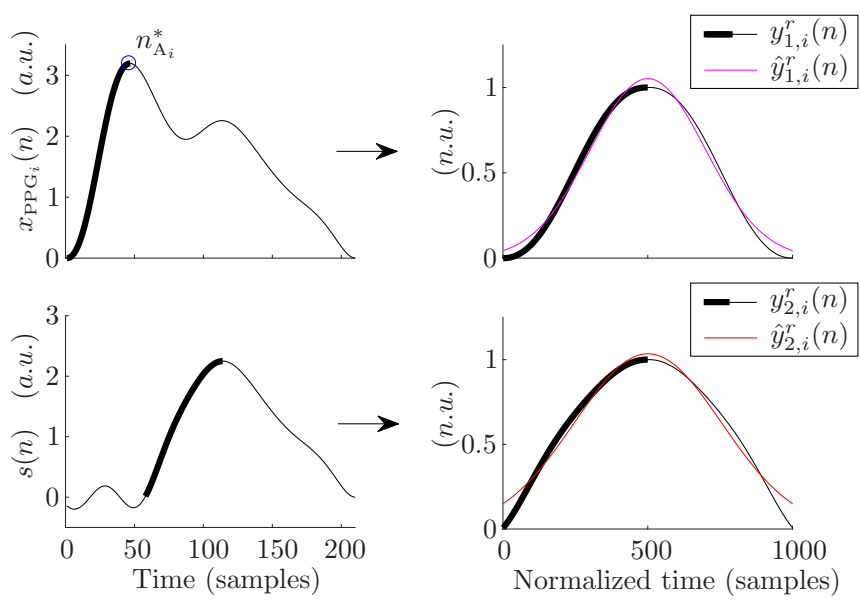

Fig. 1. Steps of the PDA technique. Upslopes which are used to fit the Gaussians are drawn with gross line.

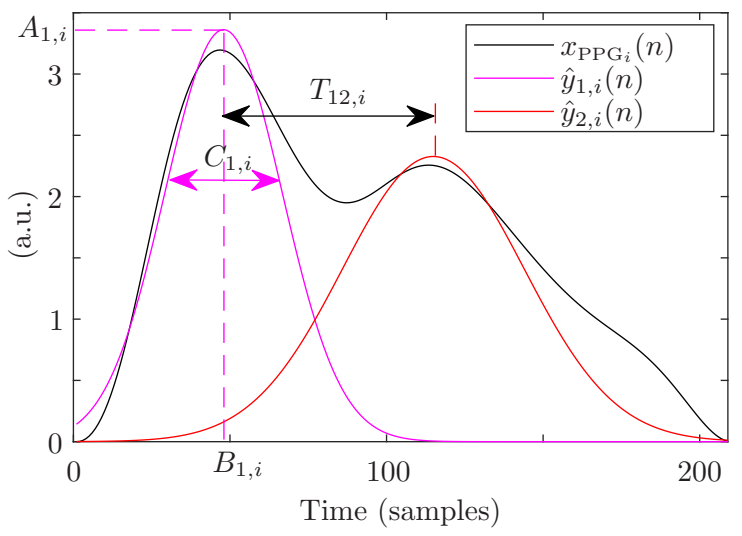

Fig. 2. PPG pulse used as example in Fig. 1 with its modeled main and first reflected waves

\section{Derived respiration signals}

Four derived respiration (DR) signals were obtained from the parameters of the modeling:

$$
\begin{aligned}
& d_{\mathrm{A} 1}^{u}(n)=\sum_{i} A_{1, i} \delta\left(n-n_{\mathrm{B}_{i}}\right) \\
& d_{\mathrm{B} 1}^{u}(n)=\sum_{i} B_{1, i} \delta\left(n-n_{\mathrm{B}_{i}}\right) \\
& d_{\mathrm{c} 1}^{u}(n)=\sum_{i} C_{1, i} \delta\left(n-n_{\mathrm{B}_{i}}\right) \\
& d_{\mathrm{T} 12}^{u}(n)=\sum_{i}\left(B_{2, i}-B_{1, i}\right) \delta\left(n-n_{\mathrm{B}_{i}}\right),
\end{aligned}
$$

where superscript " $u$ " denotes that the signals are unevenly sampled, as PPG pulses occur non-uniformly in time. The four DR signals are based on known PPG respiration-related modulations: $d_{\mathrm{A} 1}^{u}(n)$ exploits the amplitude modulation [11]; $d_{\mathrm{B} 1}^{u}(n)$ exploits the STT modulation [12]; $d_{\mathrm{C} 1}^{u}(n)$ exploits the width modulation [11]; and $d_{\mathrm{T} 12}^{u}(n)$ is related to the pulse transit time [12], which is well-known to be modulated by respiration [13]. Similarly to [11], a median-absolutedeviation outlier-rejection rule was applied, and a $4-\mathrm{Hz}-$ evenly-sampled version of each DR signal was obtained 
by cubic spline interpolation. The evenly-sampled versions of DR signals were denoted without the " $u$ " superscript, e.g., $d_{\mathrm{Al}}(n)$ is the outlier-rejected evenly sampled version of $d_{\mathrm{Al}}^{u}(n)$. Figure 3 shows an example of DR signals.
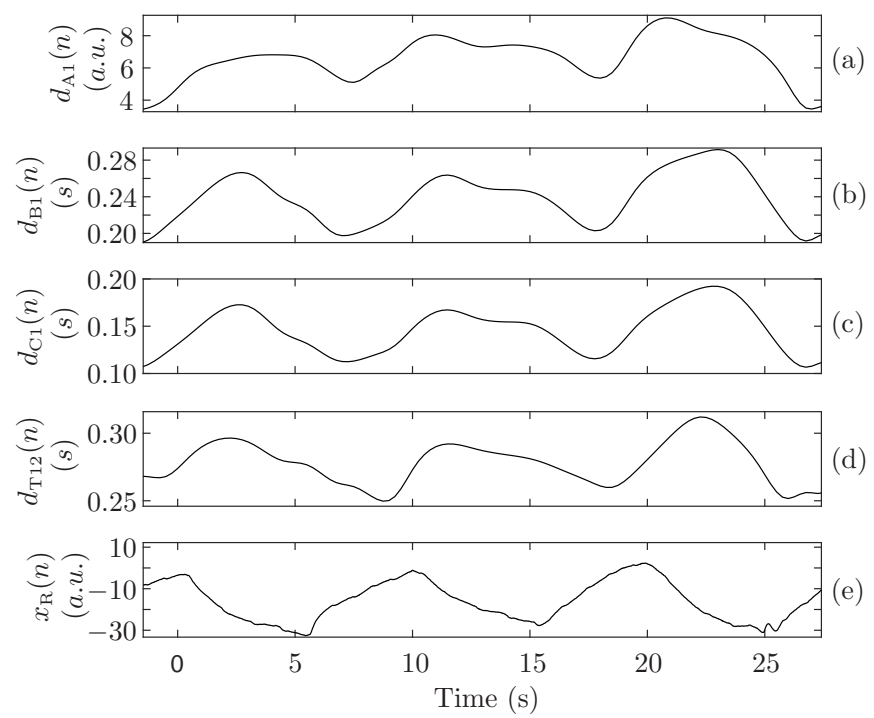

Fig. 3. Example of DR signals: a) $d_{\mathrm{A} 1}(n)$, b) $d_{\mathrm{B} 1}(n)$, c) $d_{\mathrm{C} 1}(n)$, and d) $d_{\mathrm{T} 12}(n)$; and e) the plethysmography respiratory signal.

Note that the four DR signals are obtained from one sample per pulse. This means that, similarly to all methods exploiting PPG pulse features, DR signals will not be able to follow a respiratory rate higher than half the pulse rate because of the Nyquist-Shannon sampling theorem. Thus, segments where mean pulse rate is lower than twice the requested respiratory rate were discarded for further analysis. According to this criterion, the percentage of discarded segments was $19.72 \%$ for requested respiratory rate of 0.5 $\mathrm{Hz}$, and $49.28 \%$ for respiratory rate of $0.6 \mathrm{~Hz}$. No segment was discarded for lower rates.

\section{Respiratory rate estimation}

1) From DR signals: A respiratory rate estimate was obtained for each segment from each DR signal similarly to [14]. First, the power spectral density (PSD) was estimated by a modified periodogram using a Hamming window. Then, the respiratory rate was estimated as the absolute maximum of the PSD in the respiratory band, which in this work was considered from $0.075 \mathrm{~Hz}$ to $1 \mathrm{~Hz}$.

2) From combination of $D R$ signals: Different DR signals were combined for offering a more robust respiratory rate estimation using the algorithm described in [14]. This algorithm is based on a peakness-based PSD average, which allow to take part in the average only to those PSD that have a more peaky shape (more clearly indicating an oscillation in the frequency of the maximum in the PSD).

3) From respiratory signal: The plethysmography-based respiration signal was resampled to $4 \mathrm{~Hz}$ and the respiratory rate was estimated from it by the same procedure followed with the DR signals. This respiratory rate was taken as reference in this work.

\section{E. Performance measurements}

Relative error was obtained from each estimation as:

$$
e_{\mathrm{R}}=\frac{\hat{f}_{d}-\hat{f}_{\mathrm{RES}}}{\hat{f}_{\mathrm{RES}}} \times 100
$$

where $\hat{f}_{d}(k)$ and $\hat{f}_{\mathrm{RES}}$ are the respiratory rates estimated from the evaluated DR signal, and from plethysmography-based respiration signal, respectively.

\section{RESULTS}

Table I shows median and interquartile range (IQR) of obtained $e_{\mathrm{R}}$ for each method and respiratory rate. In addition, the percentage of segments where $e_{\mathrm{R}}$ was lower than 5\% $\left(R_{e_{\mathrm{R}} \leq 5}\right)$ is also shown. According to the plethysmographybased respiration signal, subjects breathed at the requested rate with a relative error of $0.00 / 0.65 \%$ (median/IQR).

\section{TABLE I}

MEDIAN AND IQR OF $e_{\mathrm{R}}$, AND $R_{e_{\mathrm{R}} \leq 5}$, FOR EACH METHOD AND RESPIRATORY RATE. COLUMN “COMB” STANDS FOR THE COMBINED ESTIMATION.

\begin{tabular}{|c|c|c|c|c|c|c|}
\hline & & $d_{\mathrm{A} 1}(n)$ & $d_{\mathrm{B} 1}(n)$ & $d_{\mathrm{T} 12}(n)$ & $d_{\mathrm{C} 1}(n)$ & Comb \\
\hline \multirow{3}{*}{$\begin{array}{l}\vec{N} \\
\overrightarrow{0}\end{array}$} & $\operatorname{med}\left(e_{\mathrm{R}}\right)(\%)$ & 0.00 & 0.00 & 0.00 & 0.00 & 0.00 \\
\hline & $\operatorname{iqr}\left(e_{\mathrm{R}}\right)(\%)$ & 2.93 & 0.99 & 2.93 & 0.99 & 1.96 \\
\hline & $R_{e_{\mathrm{R}} \leq 5}(\%)$ & 89.53 & 93.72 & 94.24 & 91.62 & 98.95 \\
\hline \multirow{3}{*}{$\begin{array}{l}\tilde{N} \\
\text { Nै } \\
\tilde{O}\end{array}$} & $\operatorname{med}\left(e_{\mathrm{R}}\right)(\%)$ & 0.00 & 0.00 & 0.00 & 0.00 & 0.00 \\
\hline & $\operatorname{iqr}\left(e_{\mathrm{R}}\right)(\%)$ & 0.98 & 0.98 & 1.47 & 0.98 & 0.49 \\
\hline & $R_{e_{\mathrm{R}} \leq 5}(\%)$ & 90.00 & 96.19 & 80.00 & 98.57 & 99.52 \\
\hline \multirow{3}{*}{$\begin{array}{l}\text { N1 } \\
\text { m } \\
0\end{array}$} & $\operatorname{med}\left(e_{\mathrm{R}}\right)(\%)$ & 0.00 & 0.00 & 0.00 & 0.00 & 0.00 \\
\hline & $\operatorname{iqr}\left(e_{\mathrm{R}}\right)(\%)$ & 0.65 & 0.65 & 1.62 & 0.65 & 0.65 \\
\hline & $R_{e_{\mathrm{R}} \leq 5}(\%)$ & 90.95 & 92.38 & 79.05 & 92.38 & 97.62 \\
\hline \multirow{3}{*}{$\begin{array}{l}\text { Nै } \\
\stackrel{+}{0}\end{array}$} & $\operatorname{med}\left(e_{\mathrm{R}}\right)(\%)$ & 0.00 & 0.00 & -0.24 & 0.00 & 0.00 \\
\hline & $\operatorname{iqr}\left(e_{\mathrm{R}}\right)(\%)$ & 0.49 & 0.49 & 3.84 & 0.49 & 0.49 \\
\hline & $R_{e_{\mathrm{R}} \leq 5}(\%)$ & 88.15 & 91.94 & 76.78 & 94.31 & 95.73 \\
\hline \multirow{3}{*}{$\begin{array}{l}\text { Na } \\
n \\
\vdots\end{array}$} & $\operatorname{med}\left(e_{\mathrm{R}}\right)(\%)$ & 0.00 & -0.19 & -0.39 & 0.00 & -0.19 \\
\hline & $\operatorname{iqr}\left(e_{\mathrm{R}}\right)(\%)$ & 0.78 & 0.93 & 13.65 & 0.59 & 0.59 \\
\hline & $R_{e_{\mathrm{R}} \leq 5}(\%)$ & 80.70 & 81.29 & 71.35 & 87.72 & 80.12 \\
\hline \multirow{3}{*}{$\begin{array}{l}\text { N } \\
0 \\
0 \\
0\end{array}$} & $\operatorname{med}\left(e_{\mathrm{R}}\right)(\%)$ & -0.41 & -0.16 & -0.49 & -0.16 & -0.16 \\
\hline & $\operatorname{iqr}\left(e_{\mathrm{R}}\right)(\%)$ & 69.44 & 12.32 & 63.47 & 23.50 & 74.13 \\
\hline & $R_{e_{\mathrm{R}} \leq 5}(\%)$ & 57.69 & 75.00 & 59.62 & 73.08 & 69.23 \\
\hline
\end{tabular}

\section{DISCUSSION}

This paper presents a novel technique to derive respiratory rate from PPG signals, using morphological features of the PPG pulse that are known to be related by respiration. A method based on the pulse width resulted particularly interesting in [11], outperforming the other studied methods. However, it is very affected by noise and morphological changes that can be produced by many causes, e.g., a vasoconstriction event making the reflected waves closer to each other and to the main wave. PDA may help to overcome this limitation. As a first step, a PDA technique is used in this work to study how accurate it estimates the respiratory rate in non-noisy and stationary conditions.

A modification of the PDA technique described in [9] has been presented. This PDA technique allows to robustly measure different morphological features of the PPG pulse. 
In the presented technique, the waves are modeled using only their upslope considering that this upslope is much less affected by the other waves than the the rest of the wave. This physiology-related restriction remains the most relevant particularity of the presented PDA technique with respect to the other PDA techniques in literature, which usually fit a model based on a superposition of waves at once [6], [7], [8]. This restriction was imposed also in [9] for the main wave while the rest of the PPG pulse $(s(n))$ was considered to be related only to one reflected wave. In this work, the rest of the PPG pulse is considered to be formed by more than one reflected waves and so, the first reflected wave was also modeled using only its upslope. Although only 2 waves have been considered, the algorithm may be recursively applied obtaining as much reflected waves as desired if some parameters of subsequent reflected waves are interesting for the application.

The pulse amplitude $\left(A_{1}\right)$, SST $\left(B_{1}\right)$, and width $\left(C_{1}\right)$ of the main wave, as well as the time to the first reflected wave $\left(T_{12}\right)$ have been measured. DR signals have been obtained from these parameters and then, respiratory rate have been estimated from them. These estimates were compared with the respiratory rate obtained from a respiratory sensor based on plethysmography, which is a widely accepted technique. Table I shows that, in terms of median/IQR of $e_{\mathrm{R}}$, respiratory rates from 0.1 to $0.4 \mathrm{~Hz}$ were estimated by all the studied methods obtaining medians and IQRs of $e_{\mathrm{R}}$ lower than $4 \%$. This was still observed for a respiratory rate of $0.5 \mathrm{~Hz}$ with the exception of $d_{\mathrm{T} 12}(n)$. This suggest that these methods are accurately estimating respiratory rates up to 0.4 or 0.5 $\mathrm{Hz}$, depending on the method, while they are performing less accurate at respiratory rate of $0.6 \mathrm{~Hz}$. Similar behavior was observed in [14]. This may be because respirationinduced modulation probably have a less strong effect at higher respiratory rates.

Different methods obtained the same median/IQR for many cases, e.g., $d_{\mathrm{A} 1}(n), d_{\mathrm{B} 1}(n)$, and $d_{\mathrm{C} 1}(n)$ for 0.3 and 0.4 $\mathrm{Hz}(0.00 / 0.65 \%$ and $0.00 / 0.49 \%$, respectively). Therefore, it results difficult to compare them to each other in these terms. Because of this, an additional performance measure was computed: $R_{e_{\mathrm{R}} \leq 5}$. DR signal which obtained the higher $R_{e_{\mathrm{R}} \leq 5}$ in more cases was $d_{\mathrm{Cl}}(n)$ (4 cases: 0.2, 0.3, 0.4, and $0.5 \mathrm{~Hz}$ ). Furthermore, $d_{\mathrm{Cl}}(n)$ never obtained an $R_{e_{\mathrm{R}} \leq 5} 3 \%$ lower than the highest $R_{e_{\mathrm{R}} \leq 5}$ in any case. This suggest that, in terms of accuracy, $d_{\mathrm{Cl}}(n)$ should be chosen if using only one DR signal. Similar observation was made in [11], where the DR signal based on pulse width outperformed the other studied DR signals based on pulse amplitude and rate.

An additional respiratory rate estimation was obtained by combining the different DR signals by using an algorithm previously published [14]. In terms of $R_{e_{\mathrm{R}} \leq 5}$, this combination outperformed all the estimates from DR signals for respiratory rates from 0.1 to $0.4 \mathrm{~Hz}$. Furthermore, the combination never obtained the lowest $R_{e_{R} \leq 5}$ in any case. This suggest that respiratory information in the different DR signals is in part complementary. However, respiratory rate from combination did not obtain the higher $R_{e_{\mathrm{R}} \leq 5}$, indicating that combination may offer less accurate estimates than some of the used DR signals individually.

\section{CONCLUSION}

Results suggest that normal ranges of spontaneous respiratory rate $(0.1-0.5 \mathrm{~Hz})$ can be estimated from PPG pulse morphological features measured by the proposed PDA technique. These features are more robust against noise and morphological changes that usually occur in long-time recordings, than similar features measured directly over the raw PPG pulse. Further studies must be elaborated in order to compare the performance of features measured using and not using PDA during the above mentioned conditions.

\section{REFERENCES}

[1] B. Krieger, D. Feinerman, A. Zaron, and F. Bizousky, "Continuous noninvasive monitoring of respiratory rate in critically ill patients," Chest, vol. 90, no. 5, pp. 632-634, 1986.

[2] I. B. Browning, G. E. D'Alonzo, and M. J. Tobin, "Respiratory rate as an indicator of respiratory dysfunction in patients with cystic fibrosis," Chest, vol. 97, no. 6, pp. 1317-1321, 1990.

[3] A. Garde, B. Giraldo, R. Jané, T. Latshang, A. Turk, T. Hess, M. Bosch, D. Barthelmes, T. Merz, J. P. Hefti, O. Schoch, and K. Bloch, "Time-varying signal analysis to detect highaltitude periodic breathing in climbers ascending to extreme altitude," Medical \& Biological Engineering \& Computing, vol. 53, no. 8, pp. 699-712, 2015.

[4] D. Francis, K.Willson, L. C. Davies, A. J. S. Coats, and M. Piepoli, "Quantitative general theory for periodic breathing in chronic heart failure and its clinical implications," Circulation, vol. 102, p. 22142221, 2000.

[5] A. Nicolo, C. Massaroni, and L. Passfield, "Respiratory frequency during exercise: The neglected physiological measure," Frontiers in Physiology, vol. 8, pp. 1-8, 2017.

[6] M. C. Baruch, D. E. R. Warburton, S. S. D. Bredin, A. Cote, D. W. Gerdt, and C. M. Adkins, "Pulse decomposition analysis of the digital arterial pulse during hemorrhage simulation," Nonlinear Biomedical Physics, vol. 5, pp. 1-15, 2011.

[7] M. Huotari, A. Vehkaoja, K. Mtt, and J. Kostamovaara, "Photoplethysmography and its detailed pulse waveform analysis for arterial stiffness," Journal of Mechanics of Materials and Structures, vol. 44, pp. 345-362, 2011.

[8] D. Goswami, K. Chaudhuri, and J. Mukherjee, "A new two-pulse synthesis model for digital volume pulse signal analysis," Cardiovascular Engineering, vol. 10, no. 3, pp. 109-117, 2010.

[9] S. Banerjee, R. Bailón, J. Lázaro, V. Marozas, P. Laguna, and E. Gil, "A two step gaussian modelling to asses PPG morphological variability induced by psychological stress," in XLIV International Conference on Computing in Cardiology, 2017. DOI:10.22489/CinC.2017.270-035.

[10] J. Lázaro, E. Gil, J. M. Vergara, and P. Laguna, "Pulse rate variability analysis for discrimination of sleep-apnea-related decreases in the amplitude fluctuations of PPG signal in children," IEEE Journal of Biomedical and Health Informatics, vol. 18, no. 1, pp. 240-246, 2014.

[11] J. Lázaro, E. Gil, R. Bailón, A. Mincholé, and P. Laguna, "Deriving respiration from photoplethysmographic pulse width," Medical \& Biological Engineering \& Computing, vol. 51, no. 1-2, pp. 233-242, 2013.

[12] P. S. Addison, "Slope transit time (STT): A pulse transit time proxy requiring only a single signal fiducial point," IEEE Transactions on Biomedical Engineering, vol. 63, no. 11, pp. 2441-2444, 2016.

[13] C. P. Chua and C. Heneghan, "Pulse transit time-derived respiratory parameters and their variability across sleep stages," in 27th Annual Conference IEEE Engineering in Medicine and Biology, 2005, pp. 6153-6156.

[14] J. Lázaro, Y. Nam, E. Gil, P. LAguna, and K. Chon, "Respiratory rate derived from smartphone-camera-acquired pulse photoplethysmographic signals," Physiological Measurement, vol. 36, pp. 2317-2333, 2015. 